ARTICLE P 14-18

\title{
Structural mechanism of inhibitor-resistance by ERK2 mutations
}

\author{
Yun Seong Park ${ }^{\dagger}$, Myeongbin $\mathrm{Kim}^{\dagger}$ and Seong Eon Ryu* \\ Department of Bioengineering, College of Engineering, Hanyang University, Seoul 04673, Republic of Korea \\ ${ }^{*}$ Correspondence: ryuse@hanyang.ac.kr \\ ${ }^{\dagger}$ These authors contributed equally to this work.
}

Extracellular signal-regulated kinase (ERK) is a serine-threonine kinase that is involved in the regulation of cellular signals. ERK inhibitors have been developed to treat cancers with B-Raf proto-oncogene mutations. However, the use of these inhibitors in disease settings induces ERK mutations resistant to the inhibitors, which poses major difficulties in effective cancer treatment. Here, we present the crystal structures of the ERK Y36H and G37C mutants that occur in cancer cells resistant to ERK inhibitors. The structures revealed mechanisms by which these mutations confer inhibitor-resistance to ERK. The Y36H mutant structure revealed a resistance mechanism that involves rotations of the His 36 residue in the Gly-rich loop and the Tyr64 residue in the helix C, which blocks the entrance of inhibitors to the ATP-binding pocket. Furthermore, the G37C mutant structure exhibited that the mutation-induced rigidity in dihedral angles plays a major role in inducing inhibitor-resistance. Detailed structural information on the resistance mechanism suggests strategy for designing of novel inhibitors that can circumvent mutation-induced inhibitor resistance.

\section{INTRODUCTION}

Extracellular signal-regulated kinase (ERK) is a key enzyme in the mitogen-activated protein kinase (MAPK) pathway. ERK remains inactive until two amino residues within the activation loop are phosphorylated by mitogen-activated kinase (MEK) in response to extracellular growth factors. The ERK signaling pathway is involved in regulating cell proliferation, differentiation, and survival (Chaikuad et al., 2014; Goetz et al., 2014; Tong and Seeliger, 2015; Jaiswal et al., 2018; Pegram et al., 2019; Smorodinsky-Atias et al., 2020). It has been found that the tumor is resistant to ERK inhibitor treatment when the patient is continuously administered a small molecule inhibitor. It has recently been shown to induce targeted resistance to inhibitors in cancer cells in vitro as well as in vivo. And in subsequent studies, a wider variety of inhibitor-resistant mutations were identified (Smorodinsky-Atias et al., 2020).

ERK inhibition has been found to be effective in overcoming acquired resistance to BRAF/MEK inhibitors and has been used, but even if patients succeed in cancer treatment based on ERK inhibitors, patients relapse within 12 months due to acquired resistance. The patient's drug-resistant ERK showed reactivation of the MEK/ERK signaling and sustained ERK activation involving multiple mechanisms (Jaiswal et al., 2018). There have been many attempts to overcome or manage ERK resistance. Nevertheless, although inhibitors targeting ERK have had dramatic success in cancer treatment, reactivation of ERK signaling due to tolerance presents a difficult problem for many patients (Garraway and Jänne, 2012). In addition, the mechanism of obtaining ERK inhibitor resistance and signal reactivation is still unknown (Pegram et al., 2019). Therefore, understanding the regulatory mechanisms of ERK and the effect of ERK mutations on inhibitor binding is an important goal.

A significant number of ERK mutations have been reported due to the continued use of ERK inhibitors against cancer cells (Jaiswal et al., 2018; Smorodinsky-Atias et al., 2020). Since relatively little research has been conducted with respect to ERK mutations, many of their properties remain to be identified (Smorodinsky-Atias et al., 2020). For most mutations leading to inhibitor-resistances, the mechanism of action is unknown; in most cases, the effect on the structure and catalytic properties of ERKs has not been established (Goetz et al., 2014). In particular, mutations occurring at Tyr36 and Gly37, which reside in the Glyrich loop, exhibit resistance to the representative ERK2 inhibitors, Vertex-11e and SCH772984 (Brenan et al., 2016; SmorodinskyAtias et al., 2020). To understand structural mechanism of mutation-induced inhibitor resistance, we determined the crystal structures of the human ERK2 Y36H and G37C mutant proteins. The structures revealed an inhibitor-resistance mechanism that involved the conformational rearrangement of the Gly-rich loop and adjacent helix $\mathrm{C}$, and the change in loop flexibility due to the restriction of dihedral angles. This information can be exploited for designing novel inhibitors that can circumvent mutationinduced resistance.

\section{RESULTS AND DISCUSSION}

Mutation-induced loop conformation change

Structures of the inhibitor-resistant mutants Y36H and G37C of human ERK2 were determined by the molecular replacement method using the wild type rat ERK2 structure as the target. The diffraction data collection of crystals from the mutants are 
presented in Table 1. The structures were refined to 2.2 and 2.4 Å resolutions for the $\mathrm{Y} 36 \mathrm{H}$ and $\mathrm{G} 37 \mathrm{C}$ mutants, respectively (Table 1). The overall structure of the mutants was similar to that of the wild-type (Figure 1). However, significant structural differences were observed in the Gly-rich loop where residues 36 and 37 reside (Figure 1 and 2).

The structure of the $\mathrm{Y} 36 \mathrm{H}$ mutant exhibited conformational changes in the Gly-rich loop (Figure 1A). In the wild type ERK2 structure, the hydroxyphenyl ring of Tyr36 interacts with

TABLE 1 I Data collection and refinement statistics

\begin{tabular}{|c|c|c|}
\hline & Y36H & G37C \\
\hline Space group & $\mathrm{P} 2_{1}$ & $\mathrm{P} 2_{1}$ \\
\hline Resolution range $(\AA)$ & $\begin{array}{r}30.06-2.28 \\
(2.36-2.28)\end{array}$ & $\begin{array}{r}29.97-2.48 \\
(2.57-2.48)\end{array}$ \\
\hline $\begin{array}{l}\text { Unit cell dimension } \\
a, b, c(\AA) \\
\alpha, \beta, \gamma\left(^{\circ}\right)\end{array}$ & $\begin{array}{c}48.86,70.61,60.91 \\
90.00,109.65,90.00\end{array}$ & $\begin{array}{c}48.82,70.54,60.24 \\
90.00,109.40,90.00\end{array}$ \\
\hline Multiplicity & $2.8(2.1)$ & $2.9(2.6)$ \\
\hline Completeness (\%) & $94.9(85.0)$ & $94.4(96.9)$ \\
\hline$|/ \sigma|$ & $11.0(3.7)$ & $8.8(2.7)$ \\
\hline $\mathrm{R}_{\text {merge }}(\%)$ & $7.3(17.4)$ & $9.7(23.7)$ \\
\hline$R_{\text {work }} / R_{\text {free }}(\%)$ & $17.8 / 23.9$ & $18.4 / 26.6$ \\
\hline RMS in bonds $(\AA)$ / angles $\left({ }^{\circ}\right)$ & $0.008 / 0.94$ & $0.009 / 0.99$ \\
\hline Average B factor $\left(\AA^{2}\right)$ & 33.0 & 37.7 \\
\hline $\begin{array}{l}\text { Number of non-H atoms } \\
\text { macromolecules / solvent }\end{array}$ & 2832 / 102 & $2852 / 71$ \\
\hline Ramachandran favored (\%) & 95.07 & 92.51 \\
\hline Ramachandran allowed (\%) & 4.93 & 7.49 \\
\hline Ramachandran outliers (\%) & 0 & 0 \\
\hline
\end{tabular}

(A)

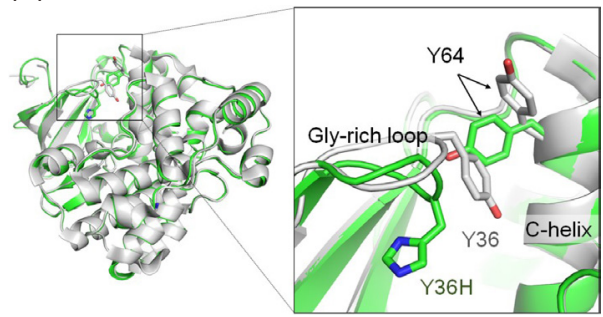

(B)

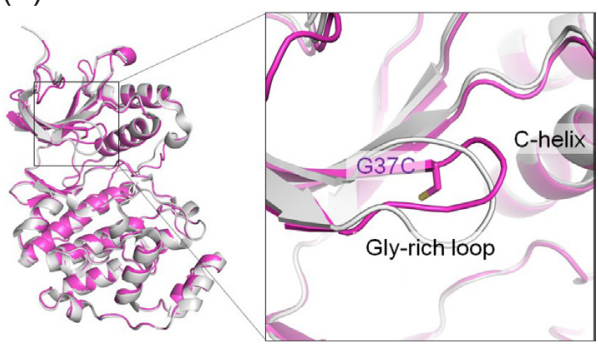

FIGURE 1 I Structure of ERK2 wild type and mutants. (A) Superposition of the wild type (PDB ID: 5UMO) and Y36H mutant structures that are in gray and green, respectively. (B) Superposition of the wild type (PDB ID: 5UMO) and G37C mutant structures that are in gray and magenta, respectively. that of Tyr64 in the helix $\mathrm{C}$, forming a $\pi-\pi$ interaction (Figure 1A). The $\mathrm{Y} 36 \mathrm{H}$ mutation, involving a side chain change from hydroxyphenyl to imidazole, disrupted the $\pi-\pi$ interaction, thereby resulting in the rotation of the imidazole side chain of His36 away from the hydroxyphenyl of Tyr64. The side chain of Tyr64 rotated and filled the empty space created by the rotation of the imidazole side chain of mutated His36 in the ERK2 Y36H mutant.

The G37C mutation introduced a thioethyl side chain in the residue, restricting the main chain dihedral angles of the residue in the favored regions of the Ramachandran plot. In the wild type structure with the Gly37 residue, the dihedral angles of the residue were $107.20^{\circ}$ and $167.10^{\circ}$ that were located in the unfavored region (Figure 3). Cys37 would not be stable with the original dihedral angles, and the G37C mutant structure exhibited dihedral angles of $-94.63^{\circ}$ and $163.21^{\circ}$ that were within the allowed regions (Figure 3 ). The change in dihedral angles in the residue 37 affected the conformation of adjacent regions, leading to rearrangement of the entire Gly-rich loop (Figure 1B). Further, the thioethyl side chain of Cys37 pointed toward the inhibitor-binding pocket (see below). The loop conformation-rearrangement also slightly shifted the position of
(A)

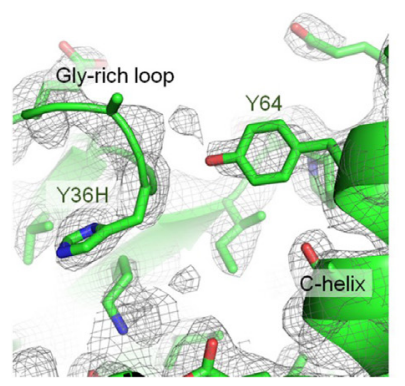

(B)

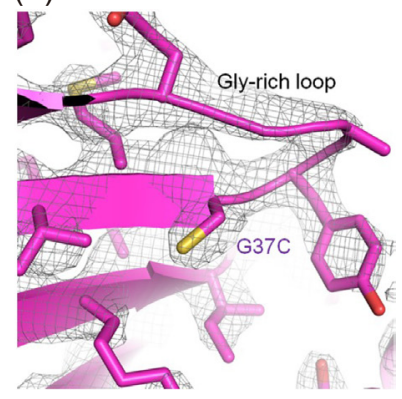

FIGURE 2 I Electron density map of ERK2 mutants. The 2Fo-Fc maps are displayed as superposed with final models. (A) The $\mathrm{Y} 36 \mathrm{H}$ mutant. (B) The G37C mutant.
(A)

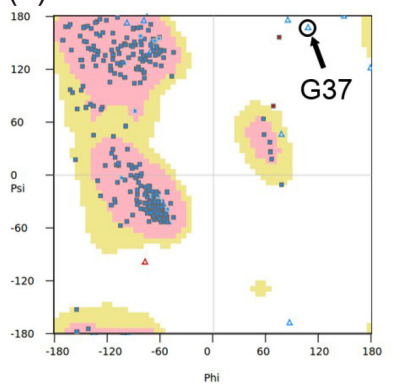

(B)

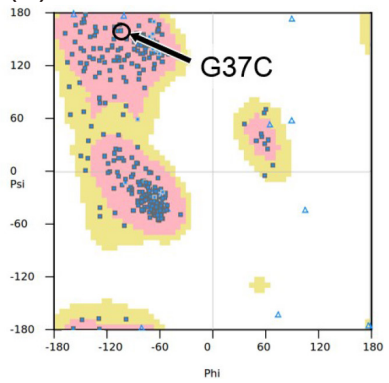

FIGURE 3 I Mutation-induced dihedral angle switch. (A) The dihediral angle position of Gly37 (black-circled blue-triangular point) in the wild type ERK2 structure (PDB ID: 5UMO) is located in the unfavored region of the Ramachandran plot. (B) The dihediral angle position of Cys37 (black-circled blue-rectangular point) in the ERK2 G37C mutant structure is located in the favored region of the Ramachandran plot. 
(A)

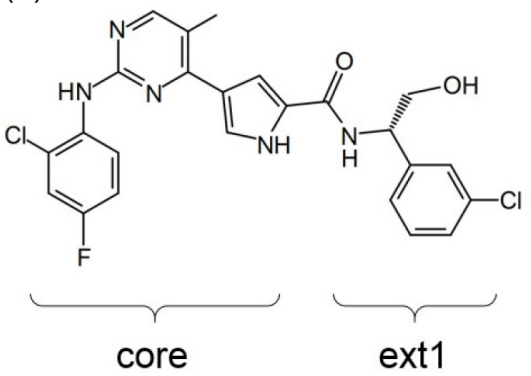

(B)

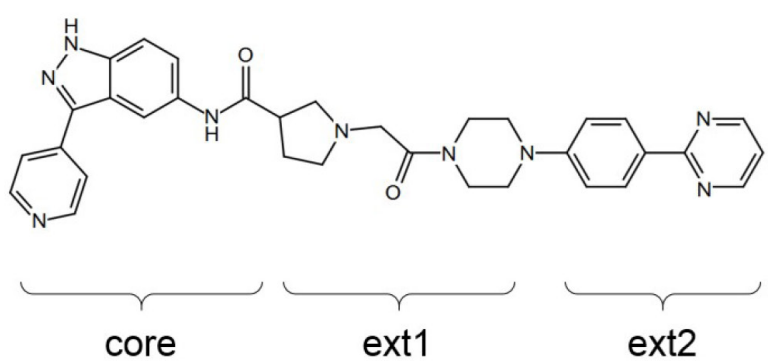

FIGURE 4 I Inhibitor structure. (A) Vertex-11e. The inhibitor parts are labeled as the core and extension 1 (ext1) regions. (B) SCH772984. The inhibitor parts are labeled as the core, extension 1 (ext1), and extension 2 (ext2) regions.

(A)

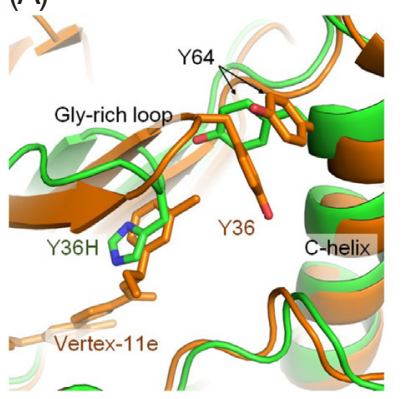

(B)

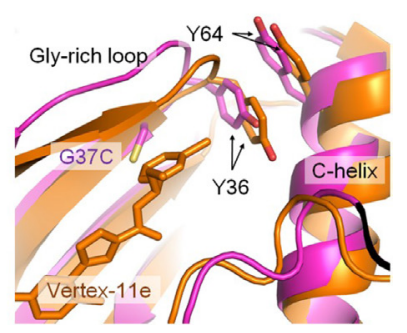

FIGURE 5 I Superposition of the ERK2 mutant structures with the Vertex11e-complexed wild type ERK2 structure (PDB ID: 6OPK). (A) The Y36H mutant is in green and the Vertex-11e-complexed wild type ERK2 is in orange. (B) The G37C mutant is in magenta and the Vertex-11e-complexed wild type ERK2 is in orange.

the hydroxyphenyl side chain of Tyr36, leading to a tighter $\pi-\pi$ interaction with the side chain of Tyr64 than that of the wild-type structure.

\section{Inhibitor resistance mechanism}

Vertex-11e and SCH772984 are representative EKR2 inhibitors that are highly potent and orally bioavailable with $\mathrm{Ki}$ values of the low nanomolar range (Aronov et al., 2009; Morris et al., 2013) (Figure 4). However, when ERK2 acquires inhibitor-resistant mutations, such as $\mathrm{Y} 36 \mathrm{H}$ and $\mathrm{G} 37 \mathrm{C}$, the inhibitors no longer exhibit enzyme inhibition. Both the inhibitors bind to the ATPbinding pocket of ERK2 (Chaikuad et al., 2014; Pegram et al., 2019). In the ERK2-inhibitor complex structure, the core regions of both the inhibitors (Figure 4) were bound to the central region of the ATP-binding pocket where the adenosine base of the ATP molecule is positioned, and extension 1 and 2 regions interact with peripheral regions of the pocket. The tip of the Gly-rich loop, involving residues 36 and 37 , provides inhibitor interactions in the peripheral regions.

The superposition of the ERK2-Vertex-11e complex with the $\mathrm{Y} 36 \mathrm{H}$ mutant indicated that the rotated imidazole side chain of His36 overlaps with the extension 1 region of the inhibitor (Figure $5 \mathrm{~A}$ ), inhibiting the binding of the inhibitor to the $\mathrm{Y} 36 \mathrm{H}$ mutant.
(A)

(B)

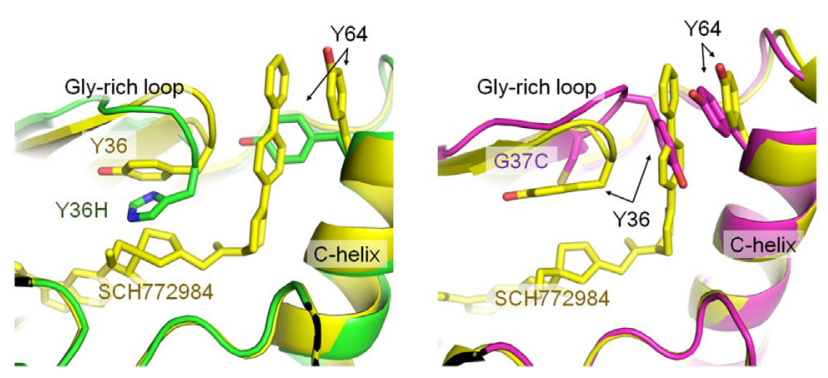

FIGURE 6 I Superposition of the ERK2 mutant structures with the SCH772984-complexed wild type ERK2 structure (PDB ID: 6OPI). (A) The $\mathrm{Y} 36 \mathrm{H}$ mutant is in green and the $\mathrm{SCH} 772984$-complexed wild type ERK2 is in yellow. (B) The G37C mutant is in magenta and the SCH772984-complexed wild type ERK2 is in yellow.

The superposition with the G37C mutant also exhibited collision of the thioethyl side chain of Cys37 to the extension 1 region of the inhibitor (Figure 5B). In the ERK2-SCH772984 complex structure, the extended piperazine-phenyl-pyrimidine (PPP) motif (extension 2 region in Figure 4) replaces the hydroxyphenyl side chain of Tyr36 to form $\pi-\pi$ interaction with the side chain of Tyr64 (Figure 6A).

However, in the $\mathrm{Y} 36 \mathrm{H}$ mutant structure, the side chain of Tyr64 rotated to the empty space created due to the rearrangement of the mutated His36, and the superposition of the ERK2$\mathrm{SCH} 772984$ complex structure with the $\mathrm{Y} 36 \mathrm{H}$ mutant structure exhibited an overlap of the PPP motif with the side chain of Tyr64. Consequently, the entrance of the PPP motif for the $\pi-\pi$ interaction was blocked. In the G37C mutant structure, the thioethyl side chain of Cys37 overlapped with the extension 1 region of $\mathrm{SCH} 772984$, inhibiting the binding of the inhibitor to the G37C mutant (Figure 6B). The lack of main chain flexibility due to addition of a side chain in residue 37 also likely contributed to the inability of inhibitor binding because the binding of the inhibitors to the ATP-binding pocket requires an induced fit of the Gly-rich loop conformation (Zhang et al., 2012). 
Implications for potent inhibitor design

Structures of the $\mathrm{Y} 36 \mathrm{H}$ and $\mathrm{G} 37 \mathrm{C}$ mutants revealed mechanisms for the inhibitor-resistance of the ERK2 mutations. In the case of the $\mathrm{Y} 36 \mathrm{H}$ mutation, the rearranged side chain of the $\mathrm{C}$-helix Tyr64 blocked access of the extension 2 region of $\mathrm{SCH} 772984$ (Figure 6). Therefore, we consider that shortening of the extension 2 region, for example, deletion of the terminal ring system, would help avoid blockage of the $\mathrm{SCH} 772984$ entrance to the ATP-binding pocket. Because the hydroxyphenyl side chain of Tyr64 is a prominent moiety in the peripheral entrance of the ATP-binding pocket, a shortened extension 2 region may yield an inhibitor with high potency and low possibility of inducing inhibitor-resistance.

The G37C mutation induced rigidity in the Gly-rich loop due to restriction of the dihedral angles. This rigidity affected the induced fit of the loop for optimal inhibitor interactions. In addition, the thioethyl side chain of the mutated Cys37 partially overlapped with extension 1 regions of both Vertex11e and SCH772984. Therefore, structural alternatives to the extension 1 region can provide optimal interactions with the wild type and mutated residues in the Gly-rich loop and holds implications in designing novel inhibitors. With the guidance of structural information of inhibitor-resistance, the rational design in combination with chemical library search can yield potent inhibitors that have less possibility of resistant mutant induction.

\section{METHODS}

Site-directed mutagenesis

The expression vector pET-28a encoding the wild-type human ERK2 full-length domain was used as a template for the polymerase chain reaction during mutagenesis. Mutations were introduced using a QuikChange sitedirected mutagenesis kit (Stratagene) and respective primers. The histidine substitution at Tyr36 was introduced using primers 5'-GCGAGGGCGCCCACGGCATGGTGTG-'3, and 5'-CACACCATGCCGTGGGC GCCCTCGC-'3 to produce the full-length ERK2 $\mathrm{Y} 36 \mathrm{H}$ mutant. The cysteine substitutions at Gly37 was introduced using primers 5 ' - GAGGGCGCCTACTGCATGGTGTGCTC - '3, and 5'-GAGCACACCATGCAGTAGGCGCCCTC-'3, to produce the full-length ERK2 G37C mutant.

\section{Expression and purification}

The recombinant vectors encoding wild type and mutated $(\mathrm{Y} 36 \mathrm{H}$ and G37C) ERK2's were used to transform an E.coli strain BL21 (DE3) competent cell. Protein overexpression in transformed cells were induced with $0.1 \mathrm{mM}$ IPTG for $16 \mathrm{~h}$ at $18^{\circ} \mathrm{C}$. The cells were harvested by centrifugation at 6,000 rpm for $10 \mathrm{~min}$ at $4^{\circ} \mathrm{C}$, washed with distilled water, then centrifuged again at $6,000 \mathrm{rpm}$ for $10 \mathrm{~min}$ and stored at $-74^{\circ} \mathrm{C}$. Harvested cells were suspended in a buffer containing $50 \mathrm{mM}$ Tris- $\mathrm{Cl}(\mathrm{pH} 7.5), 500$ $\mathrm{mM} \mathrm{NaCl}$, and $0.05 \%(\mathrm{v} / \mathrm{v}) \beta$-mercaptoethanol, and sonicated on ice. Cell lysate was separated to soluble and insoluble fractions by centrifugation at $16500 \mathrm{rpm}$ for $1 \mathrm{~h}$ at $4^{\circ} \mathrm{C}$. The His-tagged proteins were purified by affinity column (Ni-NTA, Qiagen) equilibrated with an equilibration buffer containing $50 \mathrm{mM}$ Tris$\mathrm{Cl}(\mathrm{pH}$ 7.5) and $200 \mathrm{mM} \mathrm{NaCl}$. The sample-loaded column was washed by a wash buffer containing $50 \mathrm{mM}$ Tris- $\mathrm{Cl}(\mathrm{pH} 7.5)$ and $1.0 \mathrm{M} \mathrm{NaCl}$. After the column was washed, proteins were eluted with an elution buffer containing $50 \mathrm{mM}$ Tris- $\mathrm{Cl}(\mathrm{pH}$ 7.5), $200 \mathrm{mM}$ $\mathrm{NaCl}, 500 \mathrm{mM}$ imidazole, and $5 \mathrm{mM} \beta$-mercaptoethanol. Next, the proteins were further purified by the Sephacryl S-100 gel filtration chromatography with a final buffer containing $20 \mathrm{mM}$ MOPS (pH 6.7), $150 \mathrm{mM} \mathrm{NaCl}$ and $5 \mathrm{mM} \beta$-mercaptoethanol. Purified proteins were concentrated to $30 \mathrm{mg} / \mathrm{mL}$ and stored at $-74^{\circ} \mathrm{C}$.

\section{Crystallization and diffraction data collection}

All crystallization tests were performed at $18^{\circ} \mathrm{C}$ and $4^{\circ} \mathrm{C}$ using the sitting drop vapor diffusion method in 96-well sitting drop plates, and appropriate temperatures were selected. Crystals were grown in a 1:1 ratio mixture of reservoir buffer and concentrated protein $(30 \mathrm{mg} / \mathrm{mL})$ to a final volume of $1 \mu \mathrm{L}$. Crystallization trials were performed by using the Mosquito Crystallization system (TTP Labtech) with Index (Hampton research) and Morpheus ${ }^{\circledR}$ (Molecular Dimensions) crystal screen kits. Best crystals were obtained from $100 \mathrm{mM}$ Tris- $\mathrm{Cl}(\mathrm{pH}$ 8.5), $1.35 \mathrm{M}$ ammonium sulfate, and 13\% glycerol (Y36H); $100 \mathrm{mM}$ HEPES/MOPS $(\mathrm{pH}$ 7.5), 20\% PEG 500MME, 10\% PEG 20000, 25 mM DL-glutamic acid monohydrate, $25 \mathrm{mM}$ DL-alanine, $25 \mathrm{mM}$ DL-lysine, and $25 \mathrm{mM}$ glycine (G37C). X-ray diffraction data of crystals were collected in Pohang Light Source beamlines 7A and 11C (Park et al., 2017). The crystals of ERK2 Y36H were flash-frozen using a cryo solution containing $100 \mathrm{mM}$ Tris- $\mathrm{Cl}(\mathrm{pH} 8.5), 1.35$ $\mathrm{M}$ ammonium sulfate, and $20 \%$ glycerol. In contrast, cryo solution for ERK2 G37C was $100 \mathrm{mM}$ HEPES/MOPS $(\mathrm{pH} 7.5)$, 20\% PEG 500MME, 10\% PEG 20000, 25 mM DL-glutamic acid monohydrate, $25 \mathrm{mM}$ DL-alanine, $25 \mathrm{mM}$ DL-lysine, and $25 \mathrm{mM}$ glycine. Crystals were flash-frozen in liquid nitrogen.

\section{Structure determination}

Diffraction data were processed using the program HKL2000 (Otwinowski and Minor, 1997). Data processing and merging produced 2.2 and $2.4 \AA$ resolution data sets for the $\mathrm{Y} 36 \mathrm{H}$ and G37C mutant crystals, respectively. The crystal structures were determined by the molecular replacement method using the wild type ERK2 structure (PDB code: 5UMO) as the target. For the molecular replacement solution, the program PHENIX (Liebschner et al., 2019) was used. The programs COOT (Emsley et al., 2010) and PHENIX were used for model building and refinement, respectively. $R_{\text {work }}$ and $R_{\text {free }}$ for the final model of the $\mathrm{Y} 36 \mathrm{H}$ mutant structure were 17.8 and $23.9 \%$, respectively, and $R_{\text {work }}$ and $R_{\text {free }}$ for the final model of the G37C mutant structure were 18.0 and $26.7 \%$, respectively. 


\section{DATA DEPOSITION}

Structural coordinates of human ERK2 Y36H and G37C were deposited in Protein Data Bank with codes of 7E73 and 7E75, respectively.

\section{ACKNOWLEDGEMENTS}

We thank the staff members of beamline $5 \mathrm{C}$ at the Pohang Accelerator Laboratory (PAL) for diffraction data collection. This work was supported by the biomedical technology development project, National Research Foundation, KOREA [NRF2015M3A9B5030302].

\section{CONFLICT OF INTEREST}

The authors declare no potential conflicts of interest.

Original Submission: Feb 22, 2021

Revised Version Received: Mar 16, 2021

Accepted: Mar 16, 2021

\section{REFERENCES}

Aronov, A.M., Tang, Q., Martinez-Botella, G., Bemis, G.W., Cao, J., Chen, G., Ewing, N.P., Ford, P.J., Germann, U.A., Green, J., Hale, M.R., Jacobs, M., Janetka, J.W., Maltais, F., Markland, W., et al. (2009). Structure-guided design of potent and selective pyrimidylpyrrole inhibitors of extracellular signal-regulated kinase (ERK) using conformational control. J Med Chem 52, 6362-6368.

Brenan, L., Andreev, A., Cohen, O., Pantel, S., Kamburov, A., Cacchiarelli, D., Persky, N.S., Zhu, C., Bagul, M., Goetz, E.M., Burgin, A.B., Garraway, L.A., Getz, G., Mikkelsen, T.S., Piccioni, F., et al. (2016). Phenotypic characterization of a comprehensive set of MAPK1/ERK2 missense mutants. Cell Rep 17, 1171-1183.

Chaikuad, A., Tacconi, E.M., Zimmer, J., Liang, Y., Gray, N.S., Tarsounas, M., and Knapp, S. (2014). A unique inhibitor binding site in ERK1/2 is associated with slow binding kinetics. Nat Chem Biol 10, 853-860.

Emsley, P., Lohkamp, B., Scott, W.G., and Cowtan, K. (2010). Features and development of Coot. Acta Crystallogr D Biol Crystallogr 66, 486501.

Garraway, L.A., and Jänne, P.A. (2012). Circumventing cancer drug resistance in the era of personalized medicine. Cancer Discov 2, 214-226.

Goetz, E.M., Ghandi, M., Treacy, D.J., Wagle, N., and Garraway, L.A. (2014). ERK mutations confer resistance to mitogen-activated protein kinase pathway inhibitors. Cancer Res 74, 7079-7089.

Jaiswal, B.S., Durinck, S., Stawiski, E.W., Yin, J., Wang, W., Lin, E., Moffat, J., Martin, S.E., Modrusan, Z., and Seshagiri, S. (2018). ERK mutations and amplification confer resistance to ERK-inhibitor therapy. Clin Cancer Res 24, 4044-4055.

Liebschner, D., Afonine, P.V., Baker, M.L., Bunkóczi, G., Chen, V.B., Croll, T.I., Hintze, B., Hung, L.W., Jain, S., McCoy, A.J., Moriarty, N.W., Oeffner, R.D., Poon, B.K., Prisant, M.G., Read, R.J., et al. (2019). Macromolecular structure determination using $X$-rays, neutrons and electrons: recent developments in Phenix. Acta Crystallogr D Struct Biol 75, 861-877.

Morris, E.J., Jha, S., Restaino, C.R., Dayananth, P., Zhu, H., Cooper, A., Carr, D., Deng, Y., Jin, W., Black, S., Long, B., Liu, J., Dinunzio, E., Windsor, W., Zhang, R., et al. (2013). Discovery of a novel ERK inhibitor with activity in models of acquired resistance to BRAF and MEK inhibitors. Cancer Discov 3, 742-750.

Otwinowski, Z., and Minor, W. (1997). Processing of X-ray diffraction data collected in oscillation mode. Methods Enzymol 276, 307-326.

Park, S.Y., Ha, S.C., and Kim, Y.G. (2017). The protein crystallography beamlines at the Pohang Light Source II. Biodesign 5, 30-34.

Pegram, L.M., Liddle, J.C., Xiao, Y., Hoh, M., Rudolph, J., Iverson, D.B., Vigers, G.P., Smith, D., Zhang, H., Wang, W., Moffat, J.G., and Ahn, N.G. (2019). Activation loop dynamics are controlled by conformation-selective inhibitors of ERK2. Proc Natl Acad Sci U S A 116, 15463-15468.

Smorodinsky-Atias, K., Soudah, N., and Engelberg, D. (2020). Mutations that confer drug-resistance, oncogenicity and intrinsic activity on the ERK MAP kinases-current state of the art. Cells $\mathbf{9}, 129$

Tong, M., and Seeliger, M.A. (2015). Targeting conformational plasticity of protein kinases. ACS Chem Biol 10, 190-200.

Zhang, J., Shapiro, P., and Pozharski, E. (2012). Structure of extracellular signal-regulated kinase 2 in complex with ATP and ADP. Acta Crystallogr Sect F Struct Biol Cryst Commun 68, 1434-1439. 